\title{
SPHERICAL FIBRATIONS
}

\author{
BY J. L. NOAKES
}

\begin{abstract}
In [8], [9] we extend some theorems of I. M. James and J. H. C. Whitehead on the homotopy type of spherical fibrations. Here we sketch our results and methods.
\end{abstract}

1. Introduction. Let $E_{1}, E_{2}$ be $q$-sphere Hurewicz fiberings $(q \geqslant 1)$ over the same connected finite $C W$-complex $B$. We suppose that the spaces $E_{1}, E_{2}$ have the same homotopy type $\left(E_{1} \simeq E_{2}\right)$, that $E_{2}$ has a cross-section, and that $E_{2}$ is orientable. Then by [9, Theorem 1] $E_{1}$ is also orientable. We suppose that $B$ is nilpotent [1], and let $E_{j(p)} \rightarrow B_{(p)}$ be the localization of $E_{j} \rightarrow B$ at a prime $p(j=1,2)$.

THEOREM 1. If $\operatorname{dim} B<2 q$ then, for any prime $p, E_{1(p)}$ has a cross-section.

In [3] I. M. James and J. H. C. Whitehead prove a similar result for the case where $B$ is a sphere, but where the fibre of $E_{1}, E_{2}$ is not necessarily a sphere. We comment on our proof in $\S 2$.

THEOREM 2. If $E_{1} \simeq B \times S^{q}$ where $E_{1}$ has a cross-section then $E_{1}$ is fibre homotopy trivial.

In [4] James and Whitehead take $B$ to be a sphere and prove a similar result. Our proof in [9] uses a counting argument in the spirit of [3], [7]. Comparing this proof with Theorem 1 we find that if $E_{1} \simeq B \times S^{q}$ where $\operatorname{dim} B<$ $2 q$ then $E_{1}$ is fibre homotopy trivial. It was a conjecture along these lines by I. M. James that led me to write [8], [9]. I wish to thank Professor James for telling me his conjecture.

Recall that the fibre suspension [6] $\Sigma E \rightarrow B$ of a map $\pi: E \rightarrow B$ is defined as follows. Let $\Sigma E$ be the quotient of $E \times[-1,1]$ by the relations $(e,-1) \sim\left(e^{\prime},-1\right)$ and $(e, 1) \sim\left(e^{\prime}, 1\right)$ for $\pi(e)=\pi\left(e^{\prime}\right)$. Then the projection of $\Sigma E$ takes $[e, t]$ to $\pi(e)$. We assume that $E_{2}$ has the fibre homotopy type of $\Sigma E$ for some $E$.

REMARKS. (i) If $\operatorname{dim} B<q$ then this assumption holds automatically.

(ii) If $E_{2}$ is a fibre bundle then this assumption is equivalent to the requirement that $E_{2}$ have a cross-section.

Received by the editors November 16, 1979.

AMS (MOS) subject classifications (1970). Primary 55F25, 55D10. 
THEOREM 3. If either $H^{q}(B ; Z)$ is finite or $\pi_{q} B=0$ then there is a homo topy equivalence $f: B \rightarrow B$ such that $E_{1}$ has the fibre homotopy type of the induced fibration $f^{*} E_{2}$.

EXAMPLE. Let $\Phi \subseteq \pi_{r+q} S^{q}(0<r<q-1)$ be maximal with respect to the property that if $\alpha, \beta \in \Phi$ satisfy $\alpha+\beta=0$ then $\alpha=\beta$. It follows from The orem 3 that there is a bijection from $\Phi$ onto the set of homotopy types of $q$ sphere fibrations over $S^{r+1}$.

Let $S_{j}^{q}$ be the fibre of $E_{j}(j=1,2)$.

THEOREM 4. If the pairs of spaces $\left(E_{1}, S_{1}^{q}\right),\left(E_{2}, S_{2}^{q}\right)$ have the same homc topy type then there is a homotopy equivalence $f: B \rightarrow B$ such that $E_{1}$ has the fibre homotopy type of $f^{*} E_{2}$.

In [4] James and Whitehead prove a similar result for the case where $B$ is a sphere. In [2] S. Y. Husseini considers the situation of Theorem 4, with the additional hypotheses that $\operatorname{dim} B<q$ and $\pi_{1} B=0$. Simple examples show tha $[2$, Theorem 1.1] is false as stated.

2. Methods. Our proofs of Theorems 3 and 4 turn on an elementary con struction which is difficult to describe more briefly than in [9]. However, it may be helpful to say what this construction aims to do.

Let $\pi_{j}: E_{j} \rightarrow B(j=1,2)$ be the projections, let $s_{2}$ be a cross-section of $E_{2}$ and let $\gamma: E_{2} \rightarrow E_{1}$ be a homotopy equivalence. (In the proof of Theorem 4 we take $\gamma$ to be a map of pairs.) A calculation shows that $\pi_{1} \gamma s_{2}$ is a homoto equivalence, and our construction aims to replace $\gamma$ by a homotopy equivalence $\theta^{\prime}$ so that the diagram

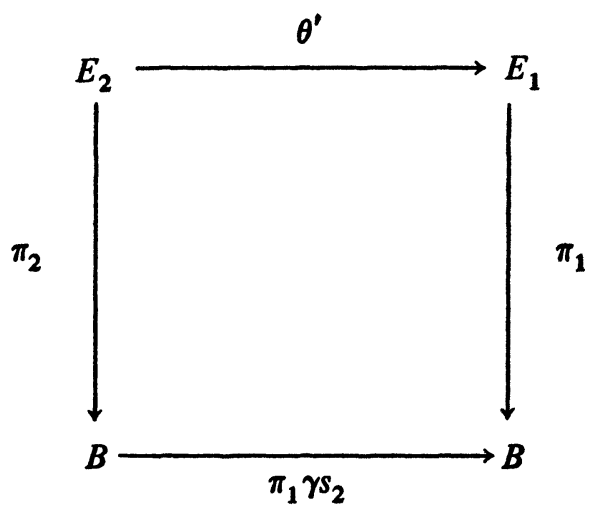

commutes.

The proof of Theorem 1 is easier to summarise. A counting argument 
taken from [7] shows that the Gysin sequences of the $E_{j(p)}$ split

$$
\begin{aligned}
& 0 \longrightarrow H^{r} B_{(p)} \stackrel{\pi_{1}^{*}}{\longrightarrow} H^{r} E_{1(p)} \stackrel{\psi_{1}}{\longrightarrow} H^{r-q_{B}} B_{(p)} \longrightarrow 0 \\
& \boldsymbol{\gamma}^{*} \downarrow \uparrow \tau^{*} \\
& 0 \longrightarrow H^{r} B_{(p)} \stackrel{\pi_{2}^{*}}{\longrightarrow} H^{r} E_{2(p)} \stackrel{\psi_{2}}{\longrightarrow} H^{r-q} B_{(p)} \longrightarrow 0
\end{aligned}
$$

for all $r$. Here $\tau$ is a homotopy inverse of $\gamma$, and we take coefficients in the ring $\mathbf{Z}_{(p)}$ of integers localized at the prime $p$. Our notation does not distinguish a map from its localization.

We choose $a_{0} \in H^{q} E_{2(p)}$ so that $\psi_{2} a_{0}$ is the identity of $H^{0} B_{(p)}$. Let $a_{2}$ $=a_{0}-\pi_{2}^{*} s_{2}^{*} a_{0}$. Then we consider two cases separately.

(1) There is no exchange at $p$ when $\psi_{1} \tau^{*} a_{2}$ is a unit of $H^{0} B_{(p)}$.

(2) There is an exchange at $p$ when $\psi_{1} \tau^{*} a_{2}$ is not a unit of $H^{0} B_{(p)}$.

When there is no exchange a computation shows that $\pi_{1} \gamma s_{2}: B \rightarrow B$ is a homotopy equivalence at the prime $p$. It quickly follows that $E_{1(p)}$ has a cross-section.

When there is an exchange we form the induced fibrations $\pi_{1}^{*} E_{1(p)}, E=$ $\iota^{*} \gamma^{*} \pi_{1}^{*} E_{1(p)}$ over $E_{1(p)}, E_{2(p)} \mid\left(B^{q-1}\right)(p)$. Here $B^{q-1}$ is the $(q-1)$-skeleton of $B$, and $\iota$ is the inclusion of $E_{2(p)} \mid\left(B^{q-1}\right)_{(p)}$ in $E_{2(p)}$.

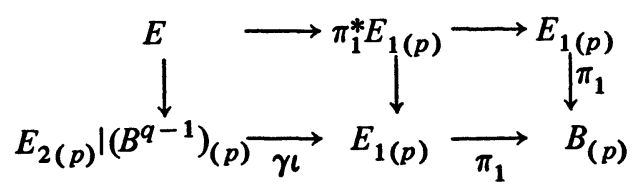

Then $\pi_{1}^{*} E_{1(p)}$ has a cross-section and therefore $E$ has a cross-section.

Next a computation using (2) and $\operatorname{dim} B<2 q$ shows that $\pi_{1} \gamma \iota$ is almost a homotopy equivalence. So the cross-section of $E$ gives rise to a cross-section of $E_{1(p)}$. For details we refer to [8].

Theorems 1,2,3 and 4 contain as special cases all the results of [4] except the case $r=q$ of $[4$, Theorem 1.6]. This exception can be dealt with from our standpoint. There remains the question of what happens when neither $E_{1}$ nor $E_{2}$ has a cross-section: for background on this we refer to [5].

\section{REFERENCES}

1. A. K. Bousfield and D. M. Kan, Homotopy limits, completions and localizations, Lecture Notes in Math., vol. 304, Springer-Verlag, Berlin and New York, 1972.

2. S. Y. Husseini, Spherical fibrations, Lecture Notes in Math., vol. 249, Springer-Verlag, Berlin and New York, 1970, pp. 107-124.

3. I. M. James and J. H. C. Whitehead, Note on fibre spaces, Proc. London Math. Soc. 4 (1954), 129-137. 
4. - The homotopy theory of sphere bundles over spheres. (I), Proc. London Math. Soc. 4 (1954), 196-218.

5. - The homotopy theory of sphere bundles over sphere. (II), Proc. LonMath. Soc. 5 (1955), 148-166.

6. 1. M. James, Overhomotopy theory, Symposia Mathematica 4 (1970), 219-229.

7. - Which fibre spaces are decomposable?, Indag. Math. 37 (1975), 385-390.

8. J. L. Noakes, Cross-sections and homotopy type (to appear).

9. - Fibrations and homotopy type (to appear).

DEPARTMENT OF MATHEMATICS, UNIVERSITY OF WESTERN AUSTRALIA, NEDLANDS, WESTERN AUSTRALIA 6009 